Laser Chem., 1999, Vol. 19, pp. 229-231

Reprints available directly from the publisher Photocopying permitted by license only
(C) 1999 OPA (Overseas Publishers Association) N.V.

Published by license under

the Harwood Academic Publishers imprint,

part of The Gordon and Breach Publishing Group.

Printed in India.

\title{
MODELING THE TRANSIENT CHANGES IN THE SORET-RESONANT RAMAN INTENSITIES OF HEMOGLOBIN DURING THE $R \rightarrow T$ TRANSITION
}

\author{
THOMAS S. RUSH III, RANJIT KUMBLE \\ and THOMAS G. SPIRO* \\ Department of Chemistry, Princeton University, Princeton, \\ New Jersey 08544, USA
}

(Received 7 April 1997)

\begin{abstract}
We have performed resonance Raman (RR) intensity calculations of the Soret-resonant Raman spectra of $\mathrm{Ni}$ and $\mathrm{Zn}$ Porphine to investigate observed core size intensity differences in the time-resolved Soret-resonant Raman (RR) spectra of hemoglobin. It was found that the metalloporphine intensities mainly derive from the expansion of the $\mathrm{C}_{\alpha}-\mathrm{C}_{m}$ and $\mathrm{C}_{\beta}-\mathrm{C}_{\beta}$ bonds in the excited state, and that the observed differences are mainly due to the larger core-sized heme having a decreased $\mathrm{C}_{\alpha}-\mathrm{C}_{m}$ force constant and larger excited state porphyrin ring expansion.
\end{abstract}

Keywords: Resonance Raman intensities; hemoglobin; core-size; Ni porphine; $\mathrm{Zn}$ porphine

Resonance Raman (RR) spectroscopy has proven to be a useful tool for the elucidation of structural and environmental properties of heme proteins. In particular, the sensitivity of the heme vibrational frequencies to changes in its ligation, oxidation and spin states, has allowed extensive application of visible RR spectroscopy to probe the properties of the heme group within the protein environment [1]. RR spectroscopy is also well suited for time-resolved protein dynamics studies, and observed transient frequency differences have successfully

\footnotetext{
*Corresponding author.
} 
been used to help elucidate heme changes which occur during hemoglobin's $\mathrm{R} \rightarrow \mathrm{T}$ transition [2]. Although the structural and environmentally induced frequency shifts in heme proteins are mostly well understood, an understanding of the even more sensitive intensity changes has not been well established. So in order to better understand the Soret-resonant heme Raman intensities, and the changes observed upon core-size expansion of the heme in the time-resolved Soretresonant Raman (RR) spectra of hemoglobin, we have modeled the $R R$ intensities of two simple metalloporphyrin model compounds. The models employed were $\mathrm{Ni}$ Porphine, with a $\mathrm{Zn}-\mathrm{N}$ distance of $\sim 1.95 \AA$, and $\mathrm{Zn}$ Porphine, with a $\mathrm{Zn}-\mathrm{N}$ distance of $\sim 2.05 \AA$.

In our investigation, we have used a simple transform theory expression for RR intensities to determine the relative influence of the electronic and vibrational structures on the observed vibrations in each of the Soret $\left(S_{0} \rightarrow S_{2}\right)$ RR spectra [3-5]:

$$
\begin{array}{r}
I_{k}\left(\omega_{L}\right) \propto \omega_{L}\left(\omega_{L}-\Omega_{K}\right)^{3}\left(\frac{1}{2}\right)\left[\left(\frac{\sqrt{\Omega_{k}}}{5.8065}\right)\left(\sum_{j=1}^{3 N-6} L_{k j}^{-1} \Delta R_{j}^{c}\right)\right]^{2} \\
\left|\phi\left(\omega_{L}\right)-\phi\left(\omega_{L}-\Omega_{k}\right)\right|^{2}
\end{array}
$$

The excited state properties of each of the metalloporphines were determined with the use of a semi-empirical INDO/s CI calculation, while the ground state structure and vibrational data were determined with a high level $a b$ initio DFT calculation. It was found that the INDO determined geometry changes predicted reasonable agreement with the experimental spectra, but they overestimated the intensity of the $\nu_{8}$ overtone in the RR spectrum of NiP. However, by comparing the experimental $\nu_{8}$ fundamental to overtone ratio, we were able to determine a scaling factor of $0.81+/-9 \%$ for the calculated geometry changes [5].

A comparison between the experimental (NiP: $\sim 0.5 \mathrm{nM}$ in $\mathrm{CS}_{2}$; $\mathrm{ZnP}: \sim 0.5 \mathrm{mM}$ in THF) and the calculated spectra are presented in Figure 1. It was found that the metalloporphine intensities mainly derive from the expansion of the $\mathrm{C}_{\alpha}-\mathrm{C}_{m}$ and $\mathrm{C}_{\beta}-\mathrm{C}_{\beta}$ bonds in the excited state. It was also determined that the changes in intensities observed in the time-resolved spectra of hemoglobin are reproduced quite well with this simple model, and are mainly due to the larger 


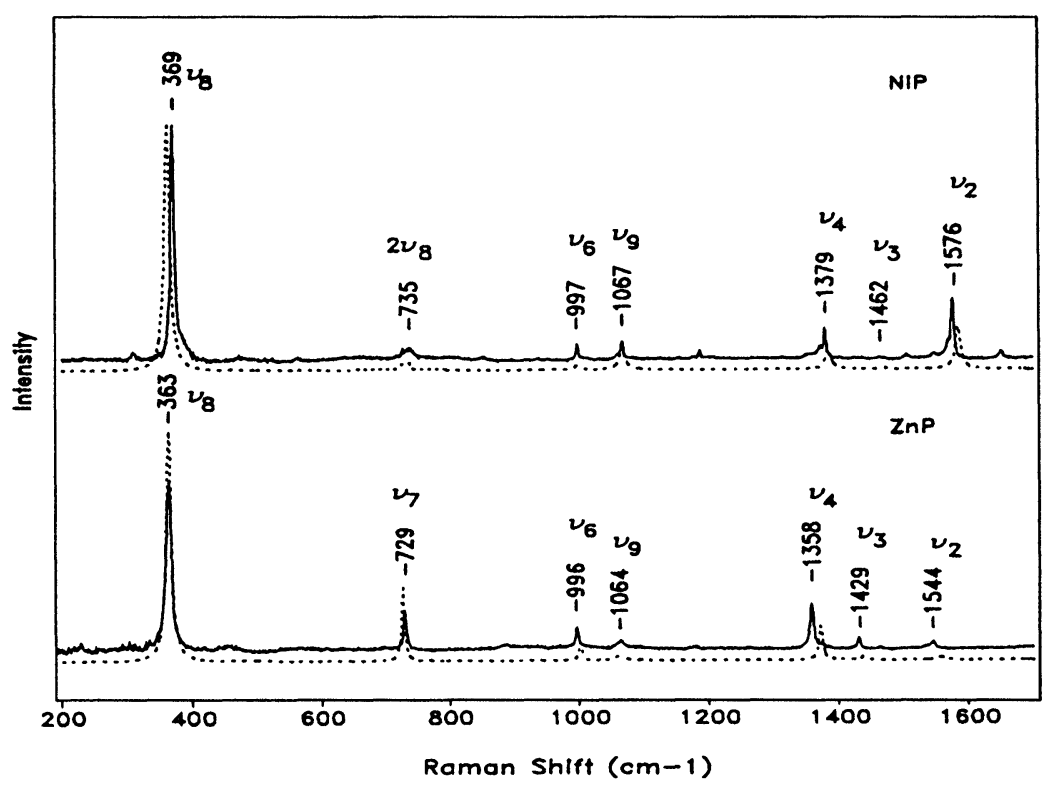

FIGURE 1 A comparison between the experimental (solid) and calculated (dashed) Soret resonant Raman spectra $(406.7 \mathrm{~nm})$ of $\mathrm{Ni}$ and $\mathrm{Zn}$ porphine.

core-sized model having a decreased $\mathrm{C}_{\alpha}-\mathrm{C}_{m}$ force constant and larger excited state core expansion. A full analysis of these spectra, and a detailed comparison of the model compound core size changes with the experimental transient hemoglobin RR intensity changes are not presented here, but will appear in an upcoming research article.

\section{References}

[1] Spiro, T. G., In: Iron Porphyrins, Part II; Lever A. and Gray H., Eds. (AddisonWesley: Reading, MA, 1983), pp. 89-160.

[2] Jayaraman, V., Rodgers, K. R., Mukerji, I. and Spiro, T. G. (1995). Science, 269, 1843.

[3] Rush III, T. and Peticolas, W. L. (1995). J. Phys. Chem., 99, 14647.

[4] Peticolas, W. L. and Rush III, T. (1995). J. Comp. Chem., 16, 126.

[5] Rush III, T., Kumble, R., Mukherjee, A., Blackwood, M. E. Jr. and Spiro, T. G. (1996). J. Phys. Chem., 100, 12076. 